\title{
Variáveis preditoras de mortalidade em pacientes com traumatismo crânio encefálico na terapia intensiva
}

\section{Predictable variables of mortality in patients with traumatic brain injury in intensive care}

Elias Ferreira Porto ${ }^{1}$, José Renato de Oliveira Leite ${ }^{2}$, Adriana Zamprônio dos Santos ${ }^{3}$

\begin{abstract}
RESUMO
Objetivo: Verificar a mortalidade, analisar as variáveis preditoras de mortalidade dos pacientes hospitalizados na unidade de terapia intensiva (UTI) por traumatismo crânio encefálico e observar a evolução dos mesmos. Método: Os dados foram coletados num hospital público de São Paulo na unidade de terapia intensiva e consistiu em avaliar o paciente diariamente e colher dados em seu prontuário. Foram estudados 20 pacientes, com idade média de 52 $\pm 19,9$ e 30 \pm 12 anos para o grupo óbito e vivos, respectivamente. Resultados: Não foi encontrada diferença significativa para os dois grupos na análise dos exames laboratoriais de sódio, potássio, cálcio, magnésio, uréia, creatinina, e na avaliação pela escala de Glasgow $(p=0,22)$. Houve maior risco de mortalidade para a presença do hematoma 2,62 (IC 1,13-6,09), infecção 3,5 (IC 1,2-5,9) dos níveis séricos de leucócitos no inicio, meio e fim para os dois grupos. Para óbito e vivos não foi encontrado diferença estatisticamente significante $(p=0,06)$. Na avaliação no qual foi comparado o leucograma inicial, meio e final para o grupo óbito separadamente, verificou-se que houve aumento significante do número de leucócitos $(p=0,0011)$. Conclusão: A infecção e a presença do hematoma aumentaram o risco de mortalidade, e a escala de Glasgow não foi um bom preditor de mortalidade.
\end{abstract}

Unitermos: Traumatismos cerebrais, Mortalidade, Unidades de Terapia Intensiva.

Citação: Porto EF, Leite JRO, Santos AZ. Variáveis preditoras de mortalidade em pacientes com traumatismo crânio encefálico na terapia intensiva. Rev Neurocienc 2007;15(1):21-27.

\section{SUMMARY}

Objective: To verify the mortality index, analyse the predicting mortality variables of hospitalised patients in the ICU due to TBI, and verify outcome. Data was collected from the ICU of a public hospital in Sao Paulo city and acquired through daily evaluation in addition to patient file note taking. Methods: Twenty patients were evaluated, average age $52 \pm 19,9$ and $30 \pm 12$ death and survival groups respectively. Results: No statistically significant differences were found between both groups in the Sodium, Potassium, Calcium, Magnesium, Urea, Creatinine and Glasgow score evaluations $(p=0.22)$. There was a higher mortality risk in the presence of haematoma 2.62 (1.13-6.09) infection 3.5 (1.2-5.9) leukocyte seric levels in the initial, middle and end stages for both groups. No statistically significant differences were found in the death or survival groups $(p=0.06)$. In the initial, middle and

\footnotetext{
Trabalho realizado no Centro Universitário Adventista de São Paulo

1 Especialista em Fisioterapia Cardiorrespiratória (InCor - HC.FMUSP), Mestre em Pneumologia e Doutorando (UNIFESP), Coordenador do Curso de Fisioterapia (UNASP).

2 Especialista em Fisioterapia Respiratória pela ASSOBRAFIR, Coordenador do Curso de Pós-graduação de Fisioterapia em UTI (UNASP) e do Curso de Especialização em Fisioterapia Cardiorrespiratória (InCor - HC.FMUSP).

3 Pós-graduanda do Curso de Especialização de Fisioterapia em UTI (UNASP).
}

Endereço para correspondência: Elias F. Porto, R. Almirante Luiz Penido Burnier, 47, apto 47 - CEP: 0586000 - São Paulo - SP

E-mail: eliasfporto@gmail.com

Trabalho recebido em 26/07/2006 • Revisado: de 27/07/2006 a 12/02/2007 • Aprovado em 13/02/2007 • Conflito de Interesse: não 
final leukocyte evaluation it was observed that there was a significant increase in the leukocyte levels for the death group alone $(p=0.0011)$. Conclusion: Infection and the presence of haematoma increased the mortality risk, and the Glasgow score was not a good mortality marker.

\section{Keywords: Brain injuries, Mortality, Intensive Care Units.}

Citation: Porto EF, Leite JRO, Santos AZ. Predictable variables of mortality in patients with traumatic brain injury in intensive care. Rev Neurocienc 2007;15(1):21-27.

\section{INTRODUÇÃO}

O Traumatismo Crânio encefálico (TCE) é definido como um tipo de agravo que induz às lesões anatômicas e comprometimento funcional envolvendo as estruturas ósseas cranianas e tecidos encefálicos. Este é classificado em leve, moderado e grave, de acordo com a escala de coma de Glasgow que é usada universalmente para quantificar o nível de lesão hemisférica cerebral em relação à gravidade e possibilidade de óbito'.

Há muito tempo existe o conhecimento de que o TCE, em seus graus de comprometimento (leve, moderado ou grave), possui diferentes resoluções clínicas. Já se identificava que existiam pacientes com TCE grave que obtinham recuperação rápida, enquanto que outros, com lesões cerebrais menores, terminavam por apresentar seqüelas importantes, e conseqüente agravamento do prognóstico?

Os parâmetros para a confecção do diagnóstico e a avaliação da gravidade nos casos de TCE não determinam o grau de traumatismo, bem como o tempo de duração das seqüelas ${ }^{3}$. Tais critérios servem única e exclusivamente para o atendimento de urgência (necessidade de intervenção cirúrgica ou de tratamento clínico imediato). Esses dados estão efetivamente mostrando que os casos de TCE não possuem critérios bem definidos para o seu entendimento, devido à ausência de fundamentos científicos. Tais critérios, por sua vez, podem ser ampliados quando verificamos que alguns dos instrumentos diagnósticos mais utilizados para avaliar o comprometimento cerebral, como o EEG, a radiografia convencional, a tomografia computadorizada (TC) e a ressonância magnética (RNM), na maioria das vezes, são incapazes de detectar alterações no TCE leve ${ }^{3,4}$.

Os pacientes vitimados de trauma que internam em Unidade de Tratamento Intensivo são sobreviventes de uma doença multissistêmica que acomete indivíduos previamente hígidos. A maioria dos casos de morte ocorre no local do evento, já os indivíduos que sobreviveram a este fato inicial, o óbito ocorre, em alguns casos, sobretudo, após a sua internação na UTI5.

Nos casos de internação, a principal indicação é a necessidade de cuidados intensivos ao paciente portador de TCE, seguida da necessidade de suporte hemodinâmico e/ou respiratório ${ }^{6}$. Pois, a maior causa de morbidade e mortalidade, dentre os vários tipos de trauma em todo o mundo, é o que afeta o crânio7.

O presente estudo ocupa-se com esse tema face à sua expressiva importância de pretender contribuir para o esclarecimento de alguns aspectos relacionados com a mortalidade após o TCE, à sua adequada avaliação e, por conseguinte, a uma melhor assistência de seus portadores.

Os objetivos foram verificar a mortalidade dos pacientes internados na unidade de terapia Intensiva, por traumatismo crânio encefálico; e identificar as variáveis preditoras de mortalidade na UTI por traumatismo crânio encefálico.

Já se encontram bem definidas algumas variáveis preditoras de mortalidade em pacientes cardiopatas, oncológicos internados em unidade de terapia intensiva, porém necessita-se de estudos mais elaborados para verificar as possíveis variáveis preditoras de mortalidade em UTI de natureza traumatológica.

É de fundamental importância para a fisioterapia a análise crítica de fatores que podem predizer a mortalidade de pacientes internados em unidade de terapia intensiva em especial nos casos de traumatismo crânio encefálico.

Nós acreditamos que este estudo poderá auxiliar na decisão e condutas médicas bem como na melhor utilização de equipamentos de uma UTI de referência para o trauma.

\section{MÉTODO}

Este é um estudo do tipo coorte, no qual foi realizada a análise dos prontuários e da evolução de 20 pacientes internados na unidade de terapia Intensiva por TCE, no período entre 02/2005 e 01/2006, independentemente das causas que motivaram o TCE. A coleta de dados foi realizada em um hospital da rede pública municipal de São Paulo, no setor da unidade de terapia intensiva, e consistia em avaliar o paciente diariamente e colher dados em seu prontuário durante o seu período de hospitalização.

O mérito deste estudo foi julgado pela comissão de ética desta instituição, o qual recebeu a sua aprovação. Para que os indivíduos participassem desse estudo era necessário possuir o seguinte critério de inclusão: estar 
internado na unidade de terapia intensiva por traumatismo crânio encefálico no período do estudo.

Não foram incluídos nesse estudo indivíduos com TCE e portadores das seguintes doenças prévias: cardiopatias graves, neoplasias, nefropatias, pneumopatias obstrutivas e/ou restritivas e esplenohepatopatias.

\section{Protocolo}

O referido estudo foi realizado através da coleta de dados durante e após a hospitalização, e do acompanhamento evolutivo dos pacientes apenas durante o período de internação na UTI.

Foram selecionados conforme o critério de inclusão, 20 pacientes internados na unidade de terapia intensiva com diagnóstico de traumatismo crânio encefálico por meio da história clínica, dos dados tomográficos e da avaliação neurológica. A partir daí, foram coletados os dados dos exames que eram realizados de rotina na UTI, ou seja, bioquímica do sangue (sódio, potássio, cálcio e magnésio), função renal (uréia e creatinina) e o leucograma de todos os pacientes. E foram analisados os exames nos seguintes momentos: início, meio e final da internação na unidade de terapia intensiva, e comparados com os valores de normalidade descritos no próprio exame.

Os exames analisados foram selecionados da seguinte forma: como início, isto é, realizado no ato da internação na UTI, como meio, ou seja, correspondente à soma da quantidade de dias de internação na UTI dividida por dois, que representava a média individual, sendo o exame selecionado àquele que mais se aproximava da mesma, desde que não tivesse grande variação de dias, motivo pelo qual permite uma avaliação mais precisa da evolução dos pacientes durante o período de internação. $E$, por conseguinte, o exame final, no qual sempre era selecionado o último exame colhido durante o período de internação na UTI.

A avaliação neurológica por meio da escala de Glasgow, considerada validada foi a primeira realizada no momento em que o paciente recebeu o pronto-atendimento, em virtude do paciente não ter sido sedado. O laudo do diagnóstico da tomografia computadorizada de crânio foi assinado pelo radiologista do próprio hospital.

Outras informações referentes aos pacientes, tais como, a causa do óbito, que era confirmada pelo atestado fornecido pelo IML, e os casos de transferência ou a alta hospitalar, foram obtidas por meio de dados contidos no prontuário.

\section{Análise estatística}

Os dados estão expressos em média e desvio padrão. Para a análise das médias repetidas de variáveis com distribuição normal foi realizado o teste de one-way
ANOVA com pós-teste de Bonferroni. E para os dados não paramétricos Kruskal-Walles testt foi utilizado o teste t não pareado para a análise das variáveis não continuas. Para estabelecer a análise das variáveis categóricas foi utilizado o Odds Ratio. Sendo considerado o $p<0,05$ como significância estatística.

\section{RESULTADOS}

A população estudada foi composta por 20 pacientes portadores de traumatismo crânio encefálico. Destes, 8 pacientes pertenciam ao grupo óbitos e 12 ao grupo vivos. No grupo óbitos, 6 eram do sexo masculino e dois do sexo feminino. Já no grupo vivos, 8 eram do sexo masculino e 4 do sexo feminino. Para

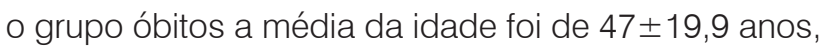
sendo que a idade mínima foi 30 e a máxima 87 anos, respectivamente. Para o grupo vivos a média de idade foi de 26,5 $\pm 12,6$ anos, sendo 17 e 58 anos as idades mínima e máxima, respectivamente $p=0,003$. A causa mais freqüente para o TCE foi a queda da própria altura, representando $62,5 \%$ dos casos. E dentre as complicações, a respiratória foi a mais freqüente durante o período de hospitalização para o grupo óbitos. Para o grupo vivos, os acidentes automobilísticos foram a causa mais freqüente para os TCE's, e nesses casos as complicações respiratórias também foram as mais evidentes (Tabelas 1,2). O tempo médio de hospitalização na unidade de terapia intensiva para o grupo óbitos foi de 27,25 dias, sendo que o tempo mínimo foi de 7 e o máximo de 56 dias, e para o grupo vivos o tempo médio de internação foi de 21,83 dias, com mínimo e máximo de 6 e 38 dias, respectivamente.

Tabela 1. Características antropométricas e clínicas do grupo óbito.

\begin{tabular}{|c|c|c|c|c|c|}
\hline Pacientes & Idade & Sexo & Glasgow & Complicações & Causas \\
\hline 2 & 35 & M & 3 & $x$ & $\begin{array}{l}\text { Queda própria } \\
\text { altura }\end{array}$ \\
\hline 6 & 49 & $\mathrm{~F}$ & 3 & $x$ & $\begin{array}{l}\text { Queda própria } \\
\text { altura }\end{array}$ \\
\hline 7 & 83 & $\mathrm{~F}$ & 9 & $x$ & $\begin{array}{l}\text { Queda própria } \\
\text { altura }\end{array}$ \\
\hline 11 & 87 & M & 15 & $x$ & Atropelamento \\
\hline 12 & 47 & M & 15 & BCP & atropelamento \\
\hline 14 & 47 & M & 7 & BCP/Sara/ITU & $\begin{array}{l}\text { Queda própria } \\
\text { altura }\end{array}$ \\
\hline 18 & 30 & M & 8 & infecção intra-operatória & Espancamento \\
\hline 20 & 39 & M & 6 & BCP/IRA & $\begin{array}{l}\text { Queda própria } \\
\text { altura }\end{array}$ \\
\hline MÉDIA & 47 & & 7,5 & & \\
\hline DP & 19,9 & & 4,3 & & \\
\hline
\end{tabular}


Foi realizada a análise dos exames de sódio, potássio, cálcio, magnésio, uréia e creatinina (Tabela 3) para o grupo vivos nos seguintes momentos, início, meio e fim da hospitalização, e não foi encontrado diferença estatisticamente significante. Bem como, na avaliação dos níveis séricos de leucócito no inicio, no meio e no final da hospitalização para dois grupos, óbitos e vivos, também não foram encontradas diferenças estatistica- mente significantes $(p=0,06)$. Já na avaliação isolada do grupo óbito, no qual foram comparados os leucogramas no inicio, meio e final da hospitalização, verificou-se que houve um aumento significativo do número de leucócitos $(p=0,0011)$. Nesse grupo o leucograma no momento inicial não apresentou desvio; já nos leucogramas dos momentos meio e final, tivemos 3 e 4 indivíduos, respectivamente, que apresentaram desvio para a esquerda.

Tabela 2. Características antropométricas e clínicas do grupo vivos.

\begin{tabular}{|c|c|c|c|c|c|}
\hline Pacientes & Idade & Sexo & Glasgow & Complicações & Causas \\
\hline 1 & 18 & $\mathrm{~F}$ & & Atelectasia/BCP & $\begin{array}{l}\text { Acidente de moto- } \\
\text { cicleta }\end{array}$ \\
\hline 3 & 23 & M & 8 & $\mathrm{BCP}$ & $\begin{array}{l}\text { Acidente automo- } \\
\text { bilístico }\end{array}$ \\
\hline 4 & 28 & $\mathrm{~F}$ & 7 & $x$ & Atropelamento \\
\hline 5 & 30 & $\mathrm{~F}$ & 8 & $\mathrm{BCP}$ & Queda do telhado \\
\hline 8 & 50 & M & 3 & $\mathrm{BCP}$ & Atropelamento \\
\hline 9 & 37 & M & 6 & Atelectasia & Queda própria altura \\
\hline 10 & 22 & M & 11 & $x$ & $\begin{array}{l}\text { Acidente automo- } \\
\text { bilístico }\end{array}$ \\
\hline 13 & 17 & M & 12 & $x$ & $\begin{array}{l}\text { Acidente automo- } \\
\text { bilístico }\end{array}$ \\
\hline 15 & 25 & $\mathrm{~F}$ & 6 & $x$ & $\begin{array}{l}\text { Acidente automo- } \\
\text { bilístico }\end{array}$ \\
\hline 16 & 40 & M & 7 & BCP/Meningite & Queda própria altura \\
\hline 17 & 58 & M & 8 & Choque hipovolêmico/BCP & Tentativa de suicídio \\
\hline 19 & 18 & M & 5 & $\mathrm{BCP}$ & $\begin{array}{l}\text { Acidente de moto- } \\
\text { cicleta }\end{array}$ \\
\hline Média & 26,5 & & 7 & & \\
\hline DP & 12,6 & & 2,7 & & \\
\hline
\end{tabular}

Tabela 3. Nível sérico de bioquímica, função renal e leucócitos para ambos os grupos.

\begin{tabular}{|c|c|c|c|c|c|c|c|c|}
\hline \multicolumn{5}{|c|}{ Grupo vivo } & \multicolumn{4}{|c|}{ Grupo óbito } \\
\hline & Inicio & Meio & Final & $p$ & Inicio & Meio & Final & $\mathrm{p}$ \\
\hline Sódio & $141 \pm 6,3$ & $138 \pm 16$ & $140 \pm 11$ & 0,58 & $142 \pm 4,1$ & $136 \pm 8,8$ & $137 \pm 12$ & 0,11 \\
\hline Potássio & $3 \pm 1,7$ & $4 \pm 1,9$ & $4 \pm 0,6$ & 0,83 & $4 \pm 1,3$ & $4 \pm 0,6$ & $5 \pm 1,4$ & 0,74 \\
\hline Cálcio & $13 \pm 2,0$ & $8 \pm 5$ & $7 \pm 2,3$ & 0,44 & $7 \pm 0,4$ & $7 \pm 021$ & $6 \pm 1$ & 0,13 \\
\hline Magnésio & $1,5 \pm 1$ & $3 \pm 2,1$ & $3 \pm 1,2$ & 0,27 & $1,4 \pm 05$ & $1,4 \pm 0,5$ & $1,8 \pm 05$ & 0,57 \\
\hline Uréia & $28 \pm 11$ & $27 \pm 12$ & $25 \pm 9,2$ & 0,83 & $32 \pm 24$ & $26 \pm 16$ & $57 \pm 37$ & 0,10 \\
\hline Creatinina & $0,9 \pm 0,2$ & $0,7 \pm 0,2$ & $0,7 \pm 0,2$ & 0,11 & $1 \pm 0,2$ & $1,5 \pm 2,1$ & $2 \pm 2,1$ & 0,56 \\
\hline Leucócitos(mil) & $11 \pm 4$ & $13,3 \pm 5,1$ & $15,6 \pm 5,2$ & 0,06 & $11,4 \pm 4,3$ & $14 \pm 6,3$ & $19 \pm 5,2$ & ${ }^{\star} 0,0011$ \\
\hline
\end{tabular}


No grupo vivos, inicialmente 1 indivíduo apresentou desvio para a esquerda, sendo que no leucograma meio 7 indivíduos, e no leucograma final 2 indivíduos tiveram desvio para esquerda, respectivamente.

Após o TCE todos os indivíduos foram submetidos à avaliação neurológica feita por meio da escala de Glasgow no período pré-admissão na unidade de terapia intensiva. Em relação a esse item, não foi encontrada diferença estatisticamente significante $(p=0,22)$ entre os grupos óbitos e vivos. Quando avaliado apenas o grupo óbito, a média foi de 6,42 44,3; sendo 3 e 15, mínimo e máximo respectivamente. Enquanto que para o grupo vivo, A média foi de 4,75 $\pm 2,7$, sendo 3 e 12, mínimo e máximo respectivamente (Figura 1).

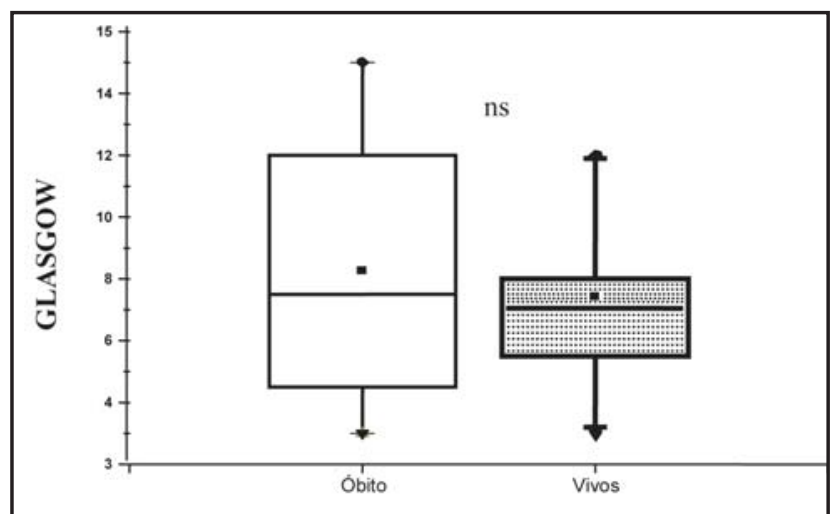

Figura 1. Avaliação neurológica por meio da escala de Glasgow para ambos os grupos.

Foi realizada a avaliação comparativa do tipo de lesão cerebral produzida pelo trauma crânio encefálico, a qual foi diagnosticada por meio da tomografia de crânio logo após a internação. Quando comparada à presença isolada de hematoma (subdural, extradural ou intracerebral) versus não ter hematoma, o risco OR foi de 1,5 (IC 0,5-4,2).

A comparação do risco de morte para pacientes que evoluíram com hematoma com os que não tiveram esse tipo de complicação foi estatisticamente significante $(p=0,02)$, evidenciando um risco de 2,62 (IC 1,12-4,9) vezes maior para o óbito do que os pacientes sem hematoma.

Quando comparamos hematoma extradural versus qualquer outro tipo de hematoma cerebral, o risco para morte também foi semelhante 1,0 (IC 0,21-4,1).

Na comparação de pacientes que não possuíam hematoma com pacientes que tinham qualquer tipo de hematoma, foi observado a presença de fator redutivo para mortalidade nos indivíduos sem hematoma OR 0,73 (IC 0,22-3,09).

Em relação aos pacientes que não possuíam hematoma quando comparados com os que desenvolveram exclusivamente Brainswealling após o trauma, também foi observado fator de redução no risco de mortalidade OR 0,50 (IC 0,12-3,7) (Figura 2).

Foi verificado que os indivíduos com leucograma alterado apresentavam risco 3,5 $(1,2-5,7)$ vezes maior de morte do que indivíduos com leucograma normal. Também foi encontrado que este mesmo grupo de indivíduos possui um risco 2,1 $(1,5-7,7)$ vezes maior de intubação oro-traqueal, quando comparado com indivíduos sem alterações infecciosas no leucograma; e para os indivíduos com infecção o risco é 3,4 $(0,75-7,7)$ vezes maior de intubação oro-traqueal quando comparado com indivíduos sem infecção (Figura 3).

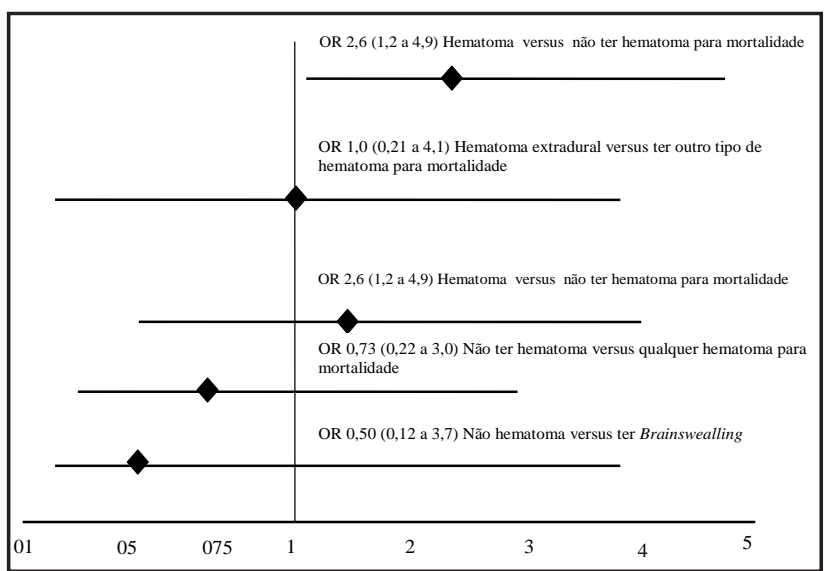

Figura 2. Odds ratio para mortalidade na presença e ausência de hematoma cerebral.

Ter infecção versus não ter infecção OR 3.5 (1.2 a 5.9)

Itubabação oro-trqueal versus não intubação oro-traqueal OR 3.4

$(1.8$ a 7.7$)$

Óbito versus vivos para intubação oro-traqueal OR 2.1 $(0.76$ a 3.8$)$

\begin{tabular}{lllllll} 
& & & & & & \\
\hline 0,50 & 0,75 & 1 & 2 & 4 & 6 & 8
\end{tabular}

Figura 3. Risco de óbito para indivíduos com e sem infecção, risco de IOT para os grupos óbitos e vivos, e risco de IOT para os indivíduos com e sem infecção.

Foi verificado que não houve transferência de pacientes para outro serviço. E todos os 12 indivíduos do grupo vivos que receberam alta para enfermaria, tiveram alta hospitalar. O tempo médio de hospitalização do grupo óbitos foi de 27,25 e do grupo vivos foi de 21,88 dias, respectivamente.

\section{DISCUSSÃO}

Dentre alguns achados deste estudo três merecem especial consideração. O primeiro, a presença de hematoma intracraniano aumentando significantemente o risco de morte em pacientes com traumatismo crânio encefálico. O segundo, o leucograma alterado que 
revelou ser um forte marcador de mortalidade para este grupo de pacientes. E o terceiro, as complicações respiratórias que foram as mais freqüentes encontradas em ambos os grupos de pacientes.

A verificação de variáveis preditoras de mortalidade em pacientes com traumatismo crânio encefálico é um método simples, que pode ser realizado por meio dos exames realizados rotineiramente em uma unidade de terapia intensiva, tornando, portanto, um método de custo baixo e de alta relevância científica. Já existem variáveis preditoras de mortalidade para cardiopatias, pneumopatias crônicas e outras afecções, já para o traumatismo crânio encefálico existem poucos estudos sobre essas possíveis variáveis.

Alguns estudos têm mostrado que o traumatismo crânio encefálico, dentre os vários tipos de trauma, é uma das maiores causas de morbidade e mortalidade em todo o mundo, sendo que a faixa etária com maior prevalência é a de adultos jovens e do sexo masculino ${ }^{7}$. A população do nosso estudo compreende uma média de idade para o grupo óbitos de $47 \pm 19$ anos, e grupo vivos de $26 \pm 5$ anos $p=0,003$, e acreditamos que esta diferença ocorreu pelo fato de que dois indivíduos deste grupo possuem idade superior a 85 anos, elevando essa média de idade. Outros 5 indivíduos deste grupo eram adultos jovens, previamente hígidos, e sem antecedentes clínicos. No que diz respeito ao grupo óbito, apesar de $100 \%$ dos indivíduos com idade superior a 85 anos pertencerem a este grupo, não podemos afirmar que a idade foi o fator de risco para mortalidade em relação ao TCE. Isso porque o tamanho da amostra analisada não é suficiente para conclusões definitivas.

Outro fato encontrado foi à predominância do sexo masculino para os dois grupos. Talvez estes dados sejam maiores nesta população pelo estilo de vida que envolve aventuras ou trabalhos com maior risco de acidente. Para esta população estudada a mortalidade foi de $40 \%$, mostrando que o TCE é um evento de alto potencial para mortalidade.

A presença de hematoma intracraniano aumentou significantemente o risco de morte nestes pacientes com traumatismo crânio encefálico. Possivelmente isto ocorreu pelo fato da hemorragia Intracraniana reduzir as funções neuroendócrinas do sistema nervoso central, sendo esta uma das principais complicações do TCE.

Alguns estudos têm mostrado que o comprometimento do sistema nervoso autônomo, ou seja, a desautonomia compromete as funções vitais, bem como a manutenção da vida, e certamente aumenta a mortalidade. Acrescenta-se ainda que nas vítimas de TCE, a deterioração da função cerebral poderá ocorrer mesmo quando a pressão intracraniana aumenta discretamente acima do limite normal. Esse fato provavelmente deve-se à perda da auto-regulação cerebrovascular, a qual torna efetivamente o cérebro mais vulnerável ${ }^{8}$. Sendo assim, a rápida deterioração da função cerebral e a morte do indivíduo ocorrem possivelmente devido aos níveis de pressão intracraniana estarem um pouco superiores aos dos valores normais ${ }^{9}$.

A hipertensão intracraniana (HIC) altera a função neurológica por meio de vários mecanismos, sendo o mais importante a diminuição do fluxo sanguíneo cerebral ${ }^{10-13}$. Este é controlado primariamente pelo fenômeno da auto-regulação cerebral, que pode ser definida como a tendência intrínseca do cérebro para manter o fluxo sanguíneo constante em resposta às moderadas variações na pressão de perfusão ${ }^{14}$. As pequenas variações da pressão intracraniana são compensadas por alterações na resistência cerebrovascular. Quando a pressão intracraniana adquire um valor próximo daquele da pressão arterial, atinge-se o ponto crítico, e o aumento prévio da pressão intracraniana determina a diminuição da pressão de perfusão e do fluxo sanguíneo cerebral. O cérebro normal pode tolerar significativa elevação da pressão intracraniana com poucas disfunções ${ }^{11}$. Nas vítimas de TCE, entretanto, a deterioração da função cerebral poderá ocorrer quando a pressão intracraniana aumenta um pouco acima do limite normal, como por exemplo, 20 a $25 \mathrm{mmHg}{ }^{15-17}$. A possível explicação para o aumento discreto da HIC seria a perda da auto-regulação cerebrovascular, tornando o cérebro mais vulnerável ao aumento da pressão intracraniana ${ }^{1}$.

Os níveis séricos de leucócitos comparados no início, meio e final da hospitalização, foram de fundamental importância na identificação de variáveis preditoras de mortalidade. A infecção é a complicação mais freqüente em pacientes graves internados na unidade de terapia intensiva, podendo ser de origem comunitária ou nasocomial. Os procedimentos invasivos, tais como, cateterização urinária, intubação traqueal, ventilação mecânica e cateteres intravasculares, são os responsáveis por grande número dessas infecções. A ação dos mediadores envolvidos no processo de infecção, resulta em hipermetabolismo, depressão da contratilidade do miocárdio, vasodilatação e diminuição da resistência vascular periférica, alteração microvascular com desregulação regional da perfusão tissular, aumento da permeabilidade vascular com extravasamento de líquido para o espaço extravascular, e consequentemente, edema intersticial e hipovolemia, distúrbio da coagulação e por fim, alterações funcionais e orgânicas. Estes mecanismos ocasionam a hipoxemia, a acidose lática e a morte celular. Como causa secundária de mortalidade para TCE estão atribuídas às complicações respiratórias. A infecção pulmonar através da liberação de mediadores químicos pelos macrófagos e neutrófilos alveolares pode ativar vários outros mediadores inflamatórios e produzir instabili- 
dade hemodinâmica e hipóxia tissular ${ }^{18}$. As infecções encontradas nos indivíduos estudados foram em sua maioria de origem respiratória, a qual foi a principal causa de morte quando relacionada ao TCE.

Acreditamos que os resultados deste estudo nos direcionam para um problema de grande relevância clínica, isto é, a infecção nosocomial, a qual está diretamente relacionada ao aumento do risco de morte. Portanto é necessário e importante que cada profissional presente nas unidades de terapia intensiva esteja alerta em relação a este fato, objetivando reduzir a incidência desse tipo de infecção.

Uma das limitações deste estudo está relacionada ao fato de não possuir os exames diários de bioquímica e leucograma, dificultando, portanto, o acompanhamento evolutivo destes pacientes.

\section{CONCLUSÃO}

Diante dos dados apresentados concluímos que, a mortalidade foi de $40 \%$ para o grupo estudado. E a existência de hematomas e o leucograma infeccioso após o TCE aumentaram o risco de mortalidade para esta população. O risco de IOT foi maior para os indivíduos com infecção pulmonar do que para os indivíduos que não tiveram leucograma infeccioso. E finalmente, a escala de Glasgow não foi um bom preditor de mortalidade para a população estudada.

\section{REFERÊNCIAS BIBLIOGRÁFICAS}

1. Contant CF, Narayan RK. Prognosis after read injury. In: Youmans JR (ed.). Neurological surgery. 4 th ed. Philadelfhia:WB Saunders, 1996, p1792-1812.

2. Manley L. A look back: concussion of the brain. J Emerg Nurs 1996; 22:33

3. Mcallister T. Mild traumatic brain injury and the postconcussive syndrome. In: Silver J, Yudofsky S, Hales R. (Eds.) Neuropsychiatry of Traumatic Brain Injury. Washington, DC: American Psychiatric Press, 1994, p357-392.

4. McClelland R. The postconcussional syndrome: a rose by any other name. J Psychosom 1996;40: 563-568

5. National Center for Health Statistics. US Department of Health and Human Services, Public Health Service. Monthy Vital Statistics Report. Ad Rep Fin Mortal Stat 1992; 43:1-76.

6. Souza PH, Mello SFL, Eifler SL. Perfil da Unidade de Trauma do Hospital do Pronto Socorro de Porto Alegre. Rev Assoc Med Bras 2002; 48-52.

7. Masini M. Perfil epidemiológico do traumatizado crânio encefálico no Distrito Federal [tese]. São Paulo: Escola Paulista de Medicina, Universidade Federal de São Paulo, 1991

8. Lassen NA. Cerebral blood flow and oxygen consumption in man. Physiol Rev 1959; 39: 183-238.

9. Adams JH, Graham DI. The relationship between ventricular fluid pressure and neuropathology raised intracranial pressure. Neuropath Appl Neurobiol 1976;2:323-232

10. Grossman RG, Turner JW, Miller JD, Rowan JO. The relationship between cortical electrical activity, cerebral perfusion pressure and cerebral blood flow duringincreased intracranial pressure. In: Langfitt TW, Mchenery LC Reivich M, Wollman H (eds). Cerebral circulation and Metabolism. Berlin:
Springer Verlag, 1975, p232-234

11. Ryder HW, Rosenauer A, Penka EJ, Espey FF, Evans JP. Failure of abnorma cerebrospinal fluid pressure to influence cerebral function. Arch Neurol Psychiatry 1953; 70: 563-586.

12. Anderson V, Catroppa C, Morse S, Haritou F. Funcional memory skills following traumatic brain injury in young children. Pediatr Rehabil 1999;3(4):159166

13. Enevoldsen EM, Cold G, Jensen FT, Malmros R. Dynamic changes in regional $\mathrm{CBF}$, intraventricular pressure, $\mathrm{CSF} \mathrm{pH}$ and lactate levels during the acute phase of head injury. J Neurosurg C 1976; 44:191-214.

14. Kalsbeek WD, McLaurin RL, Harris BS 3rd, Miller JD. The National Head and Spinal Cord Injury Survey: Major findings. J Neurosurg 1980;Suppl: S19-31.

15. Shapiro HM, Langfitt TW, Weinstein JD. Compression of cerebral vessels by intracranial hypertension: II. Morphological evidence for colapse of vessels. Acta Neurochir 1966;15:223-233.

16. Teasdale G, Rowan JO, Turner J, Grossman R, Miller JD. Cerebral perfusion failure and cortical electrical activity. In: Ingvar DH, Lassen NA (eds.). Cerebral function, metabolism and circulation. Copenhagen: Munksgaard, 1977, p430-431.

17. Traumatic brain injury. Predicting course of recovery and outcome for patients admitted to rehabilitation Arch Neurol, Jul 1994; 51: 661-670.

18. Segretti J. Nasocomial infections and secondary infections in sepsis. Crit Care Clin 1989;177-189. 